\title{
Performance analysis of the ARCHITECT anti-cyclic citrullinated peptide antibody in the diagnosis of rheumatoid arthritis
}

\author{
Sang Mee Hwang, Jeong-Ok Kim, Young-Mi Yoo, \\ Sue Shin, Jin Q Kim, Myoung Hee Park and \\ Eun Young Song* \\ Department of Laboratory Medicine, Seoul National \\ University College of Medicine and Seoul National \\ University Hospital, Seoul, Korea
}

\begin{abstract}
Background: Anti-cyclic citrullinated peptide (CCP) antibody is emerging as an important diagnostic marker for rheumatoid arthritis (RA). We evaluated the analytical and diagnostic performance of the ARCHITECT anti-CCP (Abbott Diagnostics), a new fully automated chemiluminescent microparticle immunoassay.

Methods: Serum samples from 69 patients with RA and 86 non-RA patients were used to evaluate the performance of the ARCHITECT anti-CCP assay, and the results were compared with those of EliA CCP (Phadia). The optimal cut-off value was calculated using receiver operating characteristic (ROC) curve analysis.

Results: Within-run and total imprecision (\%CV) of the ARCHITECT anti-CCP were $<6 \%$ and good linearity was observed over the claimed range. The areas under the ROC curves for the ARCHITECT anti-CCP and EliA CCP were 0.90 and 0.89 , respectively. The sensitivity and specificity were $76.8 \%$ and $95.3 \%$ for the ARCHITECT anti-CCP and $78.3 \%$ and $95.3 \%$ for EliA CCP using the manufacturer's cut-off thresholds. Both assays showed sensitivity of $84.1 \%$ and specificity of $94.2 \%$ using the optimal cut-off values.

Conclusions: The analytical performance of the ARCHITECT anti-CCP was satisfactory and diagnostic performance was comparable to that of EliA CCP. The use of optimal cutoff thresholds can yield higher sensitivity with minimal loss of specificity.

Clin Chem Lab Med 2010;48:225-30.
\end{abstract}

Keywords: analytical performance; anti-cyclic citrullinated peptide; diagnostic performance; rheumatoid arthritis.

\footnotetext{
*Corresponding author: Eun Young Song, Department of Laboratory Medicine, College of Medicine, Seoul National University, 101 Daehang-ro, Jongno-gu, Seoul, Korea Phone: + 82-2-2072-0197, Fax: + 82-2-740-8872, E-mail: eysong1@snu.ac.kr

Received August 7, 2009; accepted October 13, 2009; previously published online December 4, 2009
}

\section{Introduction}

Rheumatoid arthritis (RA) is one of the most common systemic autoimmune diseases, affecting about $1 \%$ of the population. It is characterized by chronic inflammation of the synovium, which often leads to progressive joint destruction and disability (1). The presence of rheumatoid factor (RF) is one of the criteria used by the American College of Rheumatology (ACR) for the classification of RA (2). Although the RF test has good sensitivity for RA, it lacks specificity as it can be detected in other rheumatic or inflammatory disease, and even in healthy individuals, particularly the elderly (3).

Recently, anti-cyclic citrullinated peptide (CCP) antibodies have emerged as a useful marker to predict erosive arthritis and early RA due to their high specificity (4-6). A number of tests using different cyclic citrullinated antigens have been developed with varying degrees of analytical and diagnostic performance $(7-12)$.

In the clinical laboratory, in addition to clinically confirmed diagnostic performance, application in a fully automated format, enabling for rapid testing with minimal user associated errors and good analytical performance, is important. Recently, the ARCHITECT anti-CCP assay (Abbott Laboratories, Abbott Park, IL, USA), a newly developed fully automated chemiluminescent microparticle immunoassay was introduced. The goal of this study was to evaluate the analytical (imprecision, linearity) and diagnostic (sensitivity and specificity) performance of the ARCHITECT anti-CCP assay and to compare the results of the ARCHITECT antiCCP with those from the EliA CCP test (Phadia AB, Uppsala, Sweden).

\section{Materials and methods}

\section{Materials}

A total of 155 serum samples were collected from April 2009 to September 2009 at Seoul National University Hospital. Sixty-nine serum samples were obtained from the rheumatology clinic outpatients who were diagnosed with RA based on the ACR criteria (2) (10 males and 59 females, median 59 years, age range 25-77 years). Eighty-six patients without RA, consisting of 34 patients with connective tissue diseases [17 systemic lupus erythematosus (SLE), 9 Sjögren's syndrome, 5 Behçet's disease, 3 dermatomyositis], 15 with other rheumatic disease (5 ankylosing spondylitis, 3 Takayasu's arteritis, 3 osteoarthritis, 2 polymyalgia rheumatica, 2 palindromic rheumatism); 31 patients with various viral infections (11 hepatitis B virus, 10 hepatitis $\mathrm{C}$ virus, 8 Epstein- 
Barr virus, and 2 cytomegalovirus), and six patients with other disease (2 gout, 1 relapsing polychondritis, 1 Raynaud's disease, 1 scleroderma, 1 psoriasis) (33 males and 53 females, median 50 years, age range $17-84$ years) were also collected. All serum samples were stored at $-20^{\circ} \mathrm{C}$ until analysis. This study was approved by the Institutional Review Board of Seoul National University Hospital.

\section{Methods}

Imprecision Within-run, between-day, and total coefficient of variation $(\% \mathrm{CV})$ for the ARCHITECT anti-CCP was assessed in accordance with the Clinical and Laboratory Standards Institute (CLSI) document EP15-A2 (13). Imprecision was evaluated using two serum samples (low and high antibody concentration) and one positive control material (medium antibody concentration).

Linearity The linearity evaluation was performed according to CLSI document EP6-A (14). Briefly, patient samples with low and high antibody concentrations were selected and mixed in 4:0, 3:1, $2: 2,1: 3,0: 4$ ratios to produce five concentrations that were related in linear fashion and measured in duplicate.

\section{Anti-CCP antibody assays}

All serum samples were tested with the EliA CCP assay (Phadia $\mathrm{AB}$, Uppsala, Sweden) using the ImmunoCAP 100 instrument and with the ARCHITECT anti-CCP assay (Abbott Laboratories, Abbott Park, IL, USA) using the ARCHITECT i2000. The characteristics of both assays are as follows: the EliA CCP is a fluoroenzyme immunoassay with polystyrene wells coated with original second generation synthetic CCP. The conjugate used is $\beta$-galactosidasemouse monoclonal anti-human IgG and the substrate is 4-methylumbelliferyl- $\beta$-D-galactoside. All the procedures are automated with a serum dilution of 1:100 and total incubation time of $97 \mathrm{~min}$ (30, 28, and 39 min for serum, conjugate, and substrate incubations, respectively). Six calibrators with range of $0.4-340.0 \mathrm{EliA} \mathrm{U} / \mathrm{mL}$ are used; the ARCHITECT anti-CCP assay is a chemiluminescent immunoassay that uses microparticles coated with the same second generation synthetic CCP used by the EliA CCP. The conjugate used is sulfopropyl acridinium amide-labeled polyclonal mouse antihuman IgG. Pretrigger (containing $1.32 \%$ hydrogen peroxide) and trigger (containing $0.35 \mathrm{~N}$ sodium hydroxide) solutions are added to detach the side groups of the sulfopropyl acridinium amide and make the acridinium luminescent. All procedures are automated with total incubation time of $29 \mathrm{~min}(7,18$, and $4 \mathrm{~min}$ for predilution, serum incubation, and conjugate incubation, respectively). Six calibrators with range of $0.0-200.0 \mathrm{U} / \mathrm{mL}$ are used. All measurements were performed according to the manufacturer's recommendations and performed by experienced operators who were blinded to the results of the other tests.

\section{Statistical analysis}

The sensitivity and specificity of each method was calculated using both the cut-off value recommended by the manufacturer $(\geq 5$ $\mathrm{U} / \mathrm{mL}$ for ARCHITECT anti-CCP and $>10$ EliA U/mL for the EliA $\mathrm{CCP}$ ) and the optimal cut-off value that showed the highest sum of sensitivity and specificity determined by receiver operating characteristic (ROC) curve analysis. The areas under the ROC curve (AUC) and 95\% CI were compared using the Mann-Whitney rank sum test. Correlation of the ARCHITECT anti-CCP and EliA CCP results were evaluated using Spearman's test and Bland-Altman analysis (15), after excluding results that exceeded the measurable range. Statistical analysis was performed using SPSS for Windows (version 12.0; SPSS Inc, Chicago, IL, USA). In preparing this report, the STARD guidelines (16) were taken into consideration when possible.

\section{Results}

\section{Analytical performance of ARCHITECT anti-CCP assay}

Within-run, between-day, and total CV (\%) were $1.7 \%-$ $5.5 \%, 2.4 \%-3.4 \%$, and $3.8 \%-5.9 \%$, respectively (Table 1 ). The highest imprecision was found at low antibody concentrations. Linearity was confirmed over the range of 0.95-194.6 U/mL for the ARCHITECT anti-CCP assay $(y=1.004 x-3.083, r=0.999, p<0.0001)$ (Figure 1).

\section{Diagnostic performance of ARCHITECT anti-CCP and EliA CCP assay}

The diagnostic performance for differentiating patients with RA from those without RA was evaluated using ROC curve analysis. The AUCs were 0.90 (95\% CI, 0.85-0.96) for the ARCHITECT anti-CCP and 0.89 (95\% CI, 0.82-0.95) for the EliA CCP, with no statistically significant difference between them (Figure 2).

The sensitivities, specificities, positive and negative predictive values determined using the manufacturer's and optimal cut-off values, defined as that with the highest sum of sensitivity and specificity, are shown in Table 2. Using the manufacturer's cut-off values, the sensitivity and specificity was $76.8 \%$ and $95.3 \%$, respectively, for the ARCHITECT anti-CCP and $78.3 \%$ and $95.3 \%$, respectively, for the EliA CCP. Both assays showed better diagnostic performance (sensitivity of $84.1 \%$ and specificity of $94.2 \%$ for both assays) using the optimal cut-off values $(\geq 2.6 \mathrm{U} / \mathrm{mL}$ for ARCHITECT anti-CCP and $\geq 4.8$ EliA $\mathrm{U} / \mathrm{mL}$ for EliA $\mathrm{CCP})$.

Table 1 Imprecision of the ARCHITECT anti-CCP assay.

\begin{tabular}{lclll}
\hline & Mean, U/mL & Within-run & Between-day \\
CV, \% & $\begin{array}{l}\text { Total } \\
\text { CV, \% }\end{array}$ \\
\hline Pool 1 (low) & & 3.4 & 5.5 & 2.4 \\
Positive control (medium) & 24.5 & 1.7 & 3.2 & 5.9 \\
Pool 2 (high) & 118.2 & 3.0 & 3.4 & 3.8 \\
\hline
\end{tabular}

$\mathrm{CV}$, coefficient of variation. 


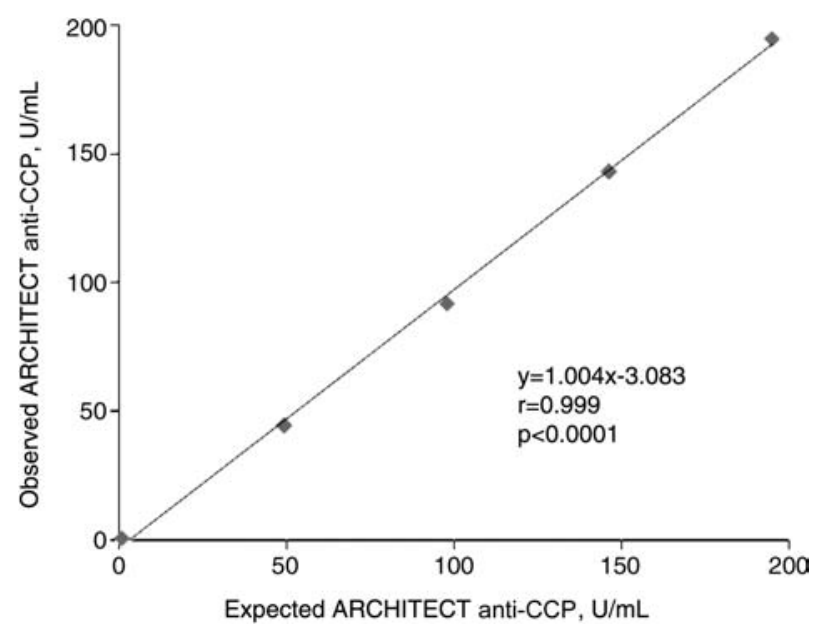

Figure 1 Linearity of the ARCHITECT anti-CCP assay. The linearity was confirmed over the range of $0.95-194.6 \mathrm{U} / \mathrm{mL}$ for the ARCHITECT anti-CCP assay $(\mathrm{y}=1.004 \mathrm{x}-3.083, \mathrm{r}=0.999$, $\mathrm{p}<0.0001)$.

Despite the high specificity of the anti-CCP assays, false positive results were observed in four patients (3 SLE and 1 palindromic rheumatism) with both assays. The distribution of anti-CCP antibody concentrations using a log scale in patients with and without RA is shown in Figure 3. The antibody concentrations for false positive results were high,

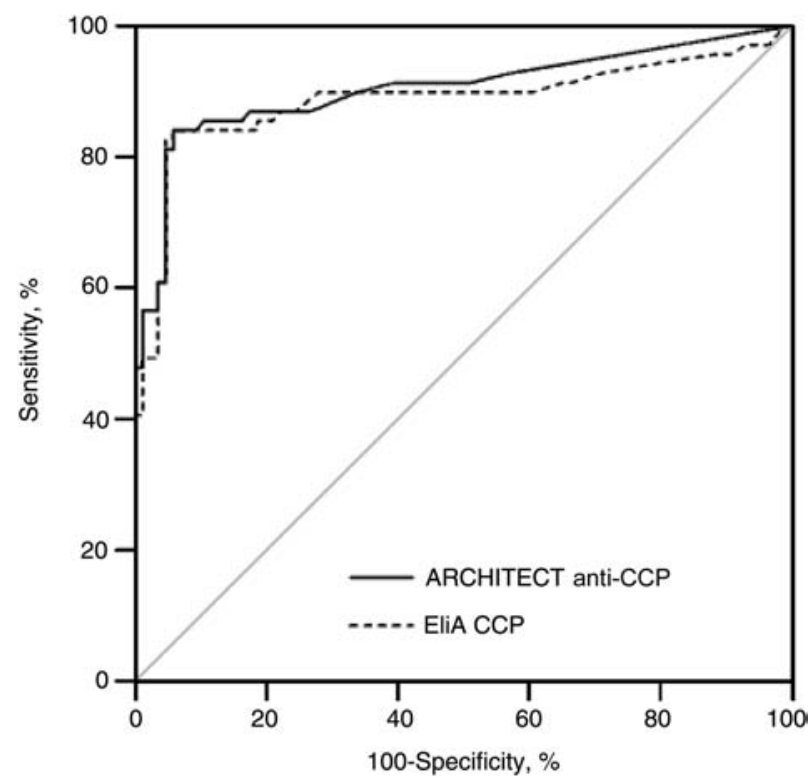

Figure 2 Receiver-operating characteristic (ROC) curves for the ARCHITECT anti-CCP and EliA CCP assay based on 69 patients with RA and 86 non-RA patients.

The area under the ROC curve (AUC) was 0.90 (95\% CI, 0.85-0.96) for the ARCHITECT anti-CCP assay and 0.89 (95\% CI, 0.82-0.95) for the EliA CCP method. With optimal cut-off values (highest sum of sensitivity and specificity, $\geq 2.6 \mathrm{U} / \mathrm{mL}$ for ARCHITECT anti-CCP and $\geq 4.8$ EliA U/mL for EliA CCP), the sensitivity and specificity were $84.1 \%$ and $94.2 \%$, respectively, for both assays.
17.4-57.8 U/mL for the ARCHITECT anti-CCP assay and 37-148 EliA U/mL for the EliA CCP method.

\section{Comparison of ARCHITECT anti-CCP and EliA CCP assay}

Quantitative results from the ARCHITECT anti-CCP (IU/mL) and EliA CCP (EliA U/mL) assays correlated significantly, with a Spearman's correlation coefficient $\left(r_{s}\right)$ of $0.860(\mathrm{p}<0.0001)$. Linear regression analysis showed a slope of 1.988 and an intercept of -0.010 (Figure 4A). A Bland-Altman bias plot showed an average difference of -26.6 (95\% CI, -131.4-78.2) between data pairs from these two assays, with the difference increasing as anti-CCP antibody concentrations increased (Figure 4B).

Comparing the qualitative results of the two assays with the manufacturer's cut-off values showed three discordant results for the RA group only. Two were negative with the ARCHITECT anti-CCP assay (2.6 and $4.4 \mathrm{U} / \mathrm{mL}$ ) but positive with the EliA CCP (10.9 and 24.2 EliA U/mL). One sample was positive with the ARCHITECT anti-CCP method (7.4 U/mL) but negative with the EliA CCP (4.8 EliA $\mathrm{U} / \mathrm{mL})$. Using the optimal cut-off values $(\geq 2.6 \mathrm{U} / \mathrm{mL}$ for the ARCHITECT anti-CCP and $\geq 4.8 \mathrm{EliA} \mathrm{U} / \mathrm{mL}$ for the EliA CCP), all results showed concordance.

\section{Discussion}

Due to the higher sensitivity and specificity compared to previous serologic markers, anti-CCP antibodies have become increasingly important for the diagnosis of RA. Since the production of the first generation anti-CCP assay in the early 2000s (7), second generation assays, using a mixture of synthetic CCPs were introduced with a significant increase in analytical sensitivity while maintaining very high specificity (96\%) (8). A number of second and third generation assays are now on the market.

A few comparative studies performed with these assays (9-12) reported better diagnostic performance for the second assays that used the original synthetic peptides, irrespective of their methodological variations, such as conjugate used and type of substrate. These findings suggest that the antigenic source is the most important variable in determining diagnostic performance. However, the analytical performance of these second generation anti-CCP assays were still variable, and sometimes unsatisfactory (9-11).

The ARCHITECT anti-CCP is a newly developed fully automated chemiluminescent microparticle immunoassay using the same synthetic peptides used in the original second generation anti-CCP assay. In this study, the ARCHITECT anti-CCP test showed good precision and linearity over the claimed range. Recent studies have reported that high antiCCP antibody concentrations at diagnosis can predict a more severe course of disease (17), and patients with high antiCCP concentrations do not respond to treatment with tumor necrosis factor- $\alpha$ inhibitors (18). These findings indicate that 
Table 2 Comparison of diagnostic performance of the ARCHITECT anti-CCP and EliA CCP assays using the manufacturer's and optimal cut-off values.

\begin{tabular}{lll}
\hline & ARCHITECT anti-CCP & EliA CCP \\
\hline Area under ROC curve (95\% CI) & $0.90(0.85-0.96)$ & $0.89(0.82-0.95)$ \\
Manufacturers' cut-off & $\geq 5 \mathrm{U} / \mathrm{mL}$ & $>10 \mathrm{EliA} \mathrm{U} / \mathrm{mL}$ \\
Sensitivity, \% (95\% CI) & $76.8(65.1-86.1)$ & $78.3(66.7-87.3)$ \\
Specificity, \% (95\% CI) & $95.3(88.5-98.7)$ & $95.3(88.5-98.7)$ \\
PPV, \% & 93.0 & 93.1 \\
NPV, \% & 83.7 & 84.5 \\
Optimal cut-off & $\geq 2.6 \mathrm{U} / \mathrm{mL}$ & $\geq 4.8 \mathrm{EliA} \mathrm{U} / \mathrm{mL}$ \\
Sensitivity, \% (95\% CI) & $84.1(73.3-91.8)$ & $84.1(73.3-91.8)$ \\
Specificity, \% (95\% CI) & $94.2(87.0-98.1)$ & $94.2(87.0-98.1)$ \\
PPV, \% & 92.1 & 92.1 \\
NPV, \% & 88.0 & 88.0 \\
\hline
\end{tabular}

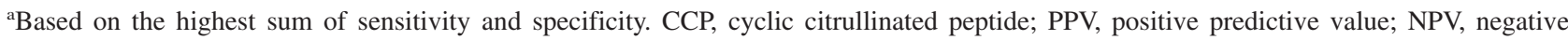
predictive value.

the analytical performance of anti-CCP as a quantitative test can be important for prognostic and therapeutic reasons.

The diagnostic performances of the ARCHITECT antiCCP and EliA CCP assays used in our study were similar to previous reports on second generation anti-CCP assays using ELISA (9-11). The EliA CCP has been reported to be a reliable second generation anti-CCP assay with good diag- nostic performance (9-11). In our study, the sensitivity and specificity of the ARCHITECT anti-CCP assay was comparable to that of the EliA CCP method when using the manufacturer's cut-off thresholds, and exactly the same with the EliA CCP using optimal cut-off values. The AUC was even slightly higher with the ARCHITECT anti-CCP (0.90) compared with the EliA CCP (0.89), although not statisti-

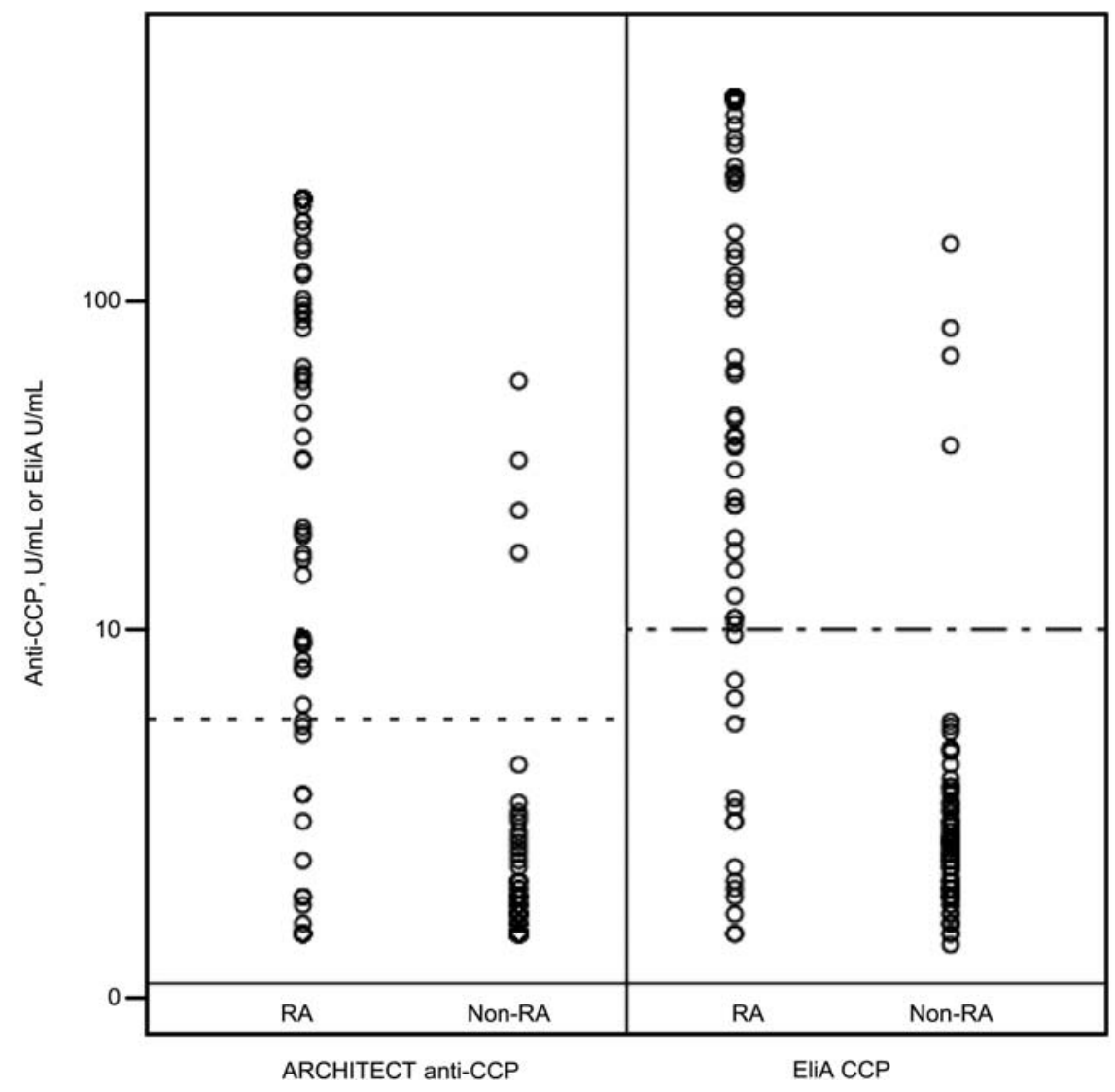

Figure 3 Distribution of anti-CCP antibody concentrations on a $\log$ scale in patients with and without RA.

The manufacturers' cut-off values (5 U/mL for ARCHITECT anti-CCP and 10 EliA U/mL for EliA CCP) are shown in dashed lines; RA, rheumatoid arthritis. 

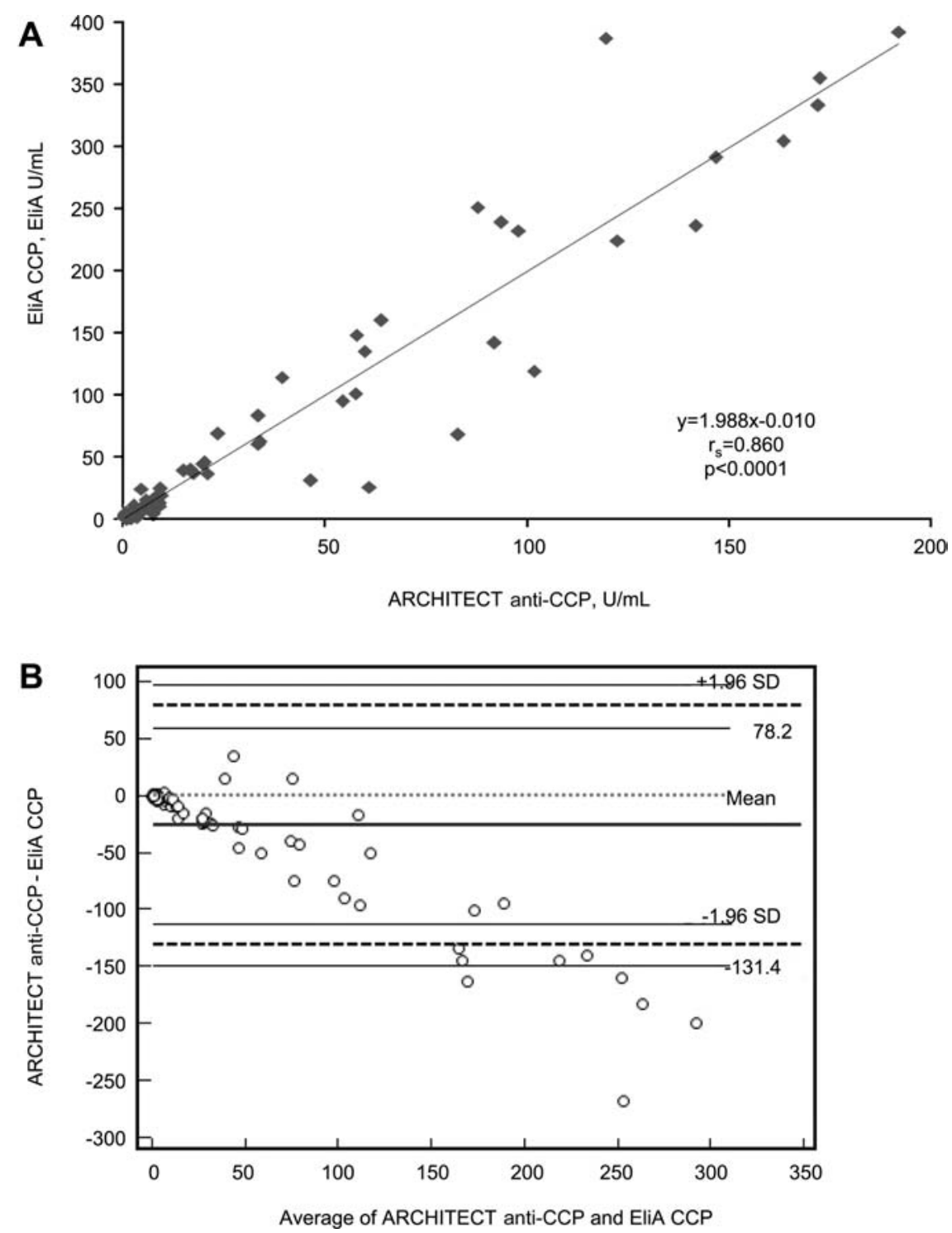

Figure 4 Correlation between the ARCHITECT anti-CCP and EliA CCP results.

(A) Scatter plot of ARCHITECT anti-CCP and EliA CCP; (B) Bland-Altman plot of ARCHITECT anti-CCP and EliA CCP.

cally significant. Adapting optimal cut-off values can be useful for the ARCHITECT anti-CCP, considering that it increases the sensitivity with only a minimal decrease in specificity. One additional false positive result with the optimal cut-off value was obtained from a SLE patient with arthralgia.

In our study, four patients in the non-RA group (3 SLE and 1 palindromic rheumatism) showed false positive results, with high anti-CCP concentrations measured using both antiCCP assays. Among them, two of three SLE patients and one patient with palindromic rheumatism had polyarthralgia, including one with radiographic changes. Recent studies have reported that the positive anti-CCP antibody results may not be false positives, but rather an important marker in diseases or symptoms related to RA. A study of 266 SLE patients showed higher anti-CCP positivity in those with arthritis compared to those without arthritis (42.1\% vs. $5.6 \%$ ) (19). In another study, among the patients who progressed from palindromic rheumatism to RA, $83 \%$ had anti-CCP antibody at the time of diagnosis (20). There were no false positive results in patients with viral infections in our study. This is in accordance with a previous report which showed that second generation assays that used the original synthetic peptides showed higher specificity in patients with viral infections, compared with other second or third generation assays that used different citrullinated antigens (11).

The correlation between the ARCHITECT anti-CCP and EliA CCP was good $\left(r_{s}=0.860\right)$ which was similar with previous reports showing good correlation between different second generation anti-CCP assays $(9,10)$. At present, the quantitative results of anti-CCP assays are not standardized, and anti-CCP concentrations obtained using different anti- 
CCP assays cannot be used interchangeably. Preparation of an international reference may allow for better comparability between different anti-CCP assays, and better use of results for diagnostic and prognostic purposes and monitoring of treatment.

For the first time to our knowledge, we evaluated the analytical and diagnostic performance of a new fully automated chemiluminescent microparticle immunoassay, the ARCHITECT anti-CCP assay. It showed good analytical performance (low imprecision and good linearity) and comparable diagnostic performance with the EliA CCP assay. The use of optimal cut-off value may yield higher sensitivity with minimal loss of specificity.

\section{Acknowledgements}

We thank Abbott Korea Ltd. Diagnostics for the generous donation of ARCHITECT anti-CCP assays.

\section{Conflict of interest statement}

Authors' conflict of interest disclosure: The authors stated that there are no conflicts of interest regarding the publication of this article. The donation of ARCHITECT anti-CCP assays played no role in the study design; in the collection, analysis, and interpretation of data; in the writing of the report; or in the decision to submit the report for publication.

Research funding: None declared.

Employment or leadership: None declared.

Honorarium: None declared.

\section{References}

1. Lee DM, Weinblatt ME. Rheumatoid arthritis. Lancet 2001;358: 903-11.

2. Arnett FC, Edworthy SM, Bloch DA, McShane DJ, Fries JF, Cooper NS, et al. The American Rheumatism Association 1987 revised criteria for the classification of rheumatoid arthritis. Arthritis Rheum 1988;31:315-24.

3. Shmerling RH, Delbanco TL. The rheumatoid factor: an analysis of clinical utility. Am J Med 1991;91:528-34.

4. Ronnelid J, Wick MC, Lampa J, Lindblad S, Nordmark B, Klareskog L, et al. Longitudinal analysis of citrullinated protein/ peptide antibodies (anti-CP) during 5-year follow-up in early rheumatoid arthritis: anti-CP status predicts worse disease activity and greater radiological progression. Ann Rheum Dis 2005; 64:1744-9.

5. Nishimura K, Sugiyama D, Kogata Y, Tsuji G, Nakazawa T, Kawano S, et al. Meta-analysis: diagnostic accuracy of anticyclic citrullinated peptide antibody and rheumatoid factor for rheumatoid arthritis. Ann Intern Med 2007;146:797-808.

6. Turesson C, Jacobsson L, Sturfelt G, Matteson E, Mathsson L, Ronnelid J. Rheumatoid factor and antibodies to cyclic citrulli- nated peptides are associated with severe extra-articular manifestations in rheumatoid arthritis. Ann Rheum Dis 2007;66: 59-64.

7. Schellekens GA, Visser H, de Jong BA, van den Hoogen FF, Hazes JM, Breedveld FC, et al. The diagnostic properties of rheumatoid arthritis antibodies recognizing a cyclic citrullinated peptide. Arthritis Rheum 2000;43:155-63.

8. van Gaalen FA, Visser H, Huizinga TW. A comparison of the diagnostic accuracy and prognostic value of the first and second anti-cyclic citrullinated peptides (CCP1 and CCP2) autoantibody tests for rheumatoid arthritis. Ann Rheum Dis 2005;64: $1510-2$.

9. Lutteri L, Malaise M, Chapelle J. Comparison of second- and third-generation anti-cyclic citrullinated peptide antibodies assays for detecting rheumatoid arthritis. Clin Chim Acta 2007; 386:76-81.

10. Coenen D, Verschueren P, Westhovens R, Bossuyt X. Technical and diagnostic performance of 6 assays for the measurement of citrullinated protein/peptide antibodies in the diagnosis of rheumatoid arthritis. Clin Chem 2007;53:498-504.

11. Bizzaro N, Tonutti E, Tozzoli R, Villalta D. Analytical and diagnostic characteristics of 11 2nd- and 3rd-generation immunoenzymatic methods for the detection of antibodies to citrullinated proteins. Clin Chem 2007;53:1527-33.

12. Mutlu N, Bicakcigil M, Tasan DA, Kaya A, Yavuz S, Ozden AI. Comparative performance analysis of 4 different anti-citrullinated protein assays in the diagnosis of rheumatoid arthritis. J Rheumatol 2009;36:491-500.

13. Clinical and Laboratory Standards Institute. User verification of performances for precision and trueness; approved guideline, 2nd ed. CLSI Document EP15-A2. Wayne, PA: Clinical and Laboratory Standards Institute, 2005.

14. Clinical and Laboratory Standards Institute. Evaluation of the linearity of quantitative measurement procedures: a statistical approach; approved guideline. CLSI Document EP6-A. Wayne PA: Clinical and Laboratory Standards Institute, 2003.

15. Bland JM, Altman DG. Statistical methods for assessing agreement between two methods of clinical measurement. Lancet 1986;327:307-10.

16. Bossuyt PM, Reitsma JB, Bruns DE, Gatsonis CA, Glasziou PP, Irwig LM, et al. The STARD statement for reporting studies of diagnostic accuracy: explanation and elaboration. Clin Chem 2003;49:7-18

17. Sihvonen S, Korpela M, Mustila A, Mustonen J. The predictive value of rheumatoid factor isotypes, anti-cyclic citrullinated peptide antibodies, and antineutrophil cytoplasmic antibodies for mortality in patients with rheumatoid arthritis. J Rheumatol 2005;32:2089-94.

18. Braun-Moscovici Y, Markovits D, Zinder O, Schapira D, Rozin A, Ehrenburg M, et al. Anti-cyclic citrullinated protein antibodies as a predictor of response to anti-tumor necrosis factor$\alpha$ therapy in patients with rheumatoid arthritis. J Rheumatol 2006;33:497-500.

19. Qing YF, Zhang QB, Zhou JG, Yuan GH, Wei J, Xing Y, et al. The detecting and clinical value of anti-cyclic citrullinated peptide antibodies in patients with systemic lupus erythematosus. Lupus 2009;18:713-7.

20. Russell AS, Devani A, Maksymowych WP. The role of anticyclic citrullinated peptide antibodies in predicting progression of palindromic rheumatism to rheumatoid arthritis. J Rheumatol 2006;33:1240-2. 